MRS Advances (C) 2016 Materials Research Society

DOI: 10.1557/adv.2016.170

\title{
Scanning Tunneling Microscopy of Atomic Scale Phonon Standing Waves in Quasi-freestanding WSe $\mathrm{W}_{2}$ Monolayers
}

\author{
Igor Altfeder $^{1 *}$, Sarah M. Eichfeld ${ }^{2}$, Rachel D. Naguy ${ }^{1}$, Joshua A. Robinson ${ }^{2}$ and Andrey A. \\ Voevodin ${ }^{1}$ \\ ${ }^{1}$ Nanoelectronic Materials Branch, Air Force Research Laboratory, Wright Patterson AFB, OH \\ 45433, USA \\ ${ }^{2}$ Department of Materials Science and Engineering and The Center for Two-Dimensional and \\ Layered Materials, The Pennsylvania State University, University Park, PA 16802, USA
}

\begin{abstract}
Using scanning tunneling microscopy (STM) we observed atomic scale interference patterns on quasi-freestanding $\mathrm{WSe}_{2}$ islands grown on top of graphene. The bias-independent double atomic size periodicity of these patterns and the sharp Brillouin zone edge revealed by 2D STM Fourier analysis indicate formation of optical phonon standing waves due to scattering on intercalating defects supporting these islands. Standing wave patterns of both synchronized and non-synchronized optical phonons, corresponding to resonant and non-resonant phonon scattering regimes, were experimentally observed. We also found the symmetry breaking effect for individual phonon wave packets, one of the unique features distinguishing phonon standing waves. We show that vibrational and electronic anharmonicities are responsible for STM detection of these patterns. A significant contribution to the interference contrast arises from quantum zero-point oscillations.
\end{abstract}

\section{INTRODUCTION}

The unusual behavior of phonons in nanomaterials manifests in such phenomena as spontaneous ripples on graphene [1], inelastic Friedel oscillations [2], phonon tunneling in subnanometer gaps [3], and phonon interference in quantum wells [4]. Experimental observation of atomic scale phonon standing waves that will be described here opens new page in this rapidly developing research field. A large number of theoretical and experimental publications $[5,6]$ have confirmed that phonons can be efficiently scattered by individual lattice defects and

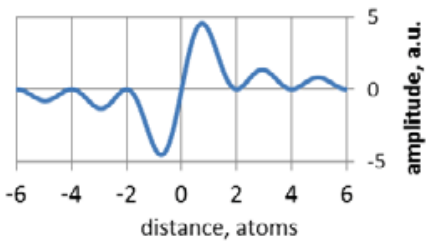

Figure 1. Simulated interference pattern for dispersionless 1D optical phonons. The scattering center is located at $x=0$. The pattern profile: $\left(k_{0} x\right)^{-1}\left(1-\cos k_{0} x\right)$, where $k_{0}=\pi / a$. Vertical axis periodically reverses sign because of oscillations. 
individual intercalating atoms. To understand what periodicities are anticipated from defectinduced phonon standing wave patterns, in the Figure 1 we show the simulated interference wave packet for a simplest case of nearly-dispersionless one-dimensional optical phonons. The construction of this curve takes into account (a) significant phonon scattering probability and (b) summation over all Brillouin zone (BZ). In addition, due to narrow oscillator frequency distribution, phase synchronization of all $\omega(k)$ modes caused by defect-induced coupling between them has to be taken into account $[7,8]$. The double atomic size $(2 a)$ periodic interference fringes on this curve arise from the wavelength cutoff at the BZ edge. It is anticipated that in STM experimental setup these standing waves will be detected as static $2 a$ periodic interference patterns due to "rectification" effect caused by electron-phonon coupling [9] and phonon anharmonicities [10]. Although ideally this phenomenon would require isolated free standing atomic layers, as we will later show, using STM it can be observed in quasifreestanding $\mathrm{WSe}_{2}$ [11] monolayers on graphene. The presence of several nearly-dispersionless, $\Delta \omega / \omega \approx 0.05$, optical phonon branches at $250 \mathrm{~cm}^{-1}$ [11] makes $\mathrm{WSe}_{2}$ an interesting model system for study of this effect.

\section{EXPERIMENTAL DETAILS}

The $\mathrm{WSe}_{2}$ /graphene samples for our study were prepared using metal-organic chemical vapor deposition (MOCVD) technique described in earlier publications $[12,13]$. The presence of $\mathrm{WSe}_{2}$ nanostructures on surfaces of studied samples was confirmed by ambient atomic force microscopy. The sample was then transferred into the ultra-high vacuum STM system (model UHV300 from RHK Technology) with base pressure $7 \times 10^{-11}$ Torr. Before STM measurements, the samples were in situ annealed to $350^{\circ} \mathrm{C}$ for few hours in order to eliminate the adsorbed water from their surfaces. The commercial Pt-Ir STM tip (from Bruker Corp.) in situ cleaned using electron beam heating technique has been used. The STM measurements were performed at room temperature.

The STM measurements revealed two types of $\mathrm{WSe}_{2}$ nanostructures: multi-layered spiral pyramids and atomically flat islands. The typical STM image of $\mathrm{WSe}_{2}$ film is shown in the

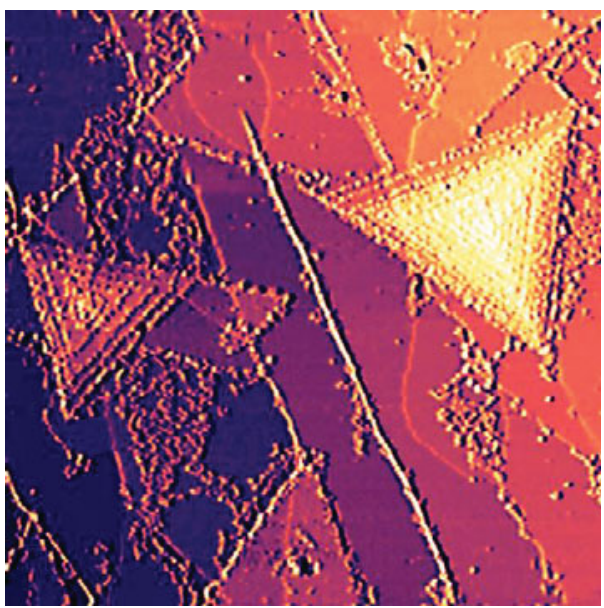

Figure 2. The $0.65 \times 0.65 \mu \mathrm{m}^{2} \mathrm{STM}$ image of $\mathrm{WSe}_{2}$ film grown by MOCVD on graphene. 
Figure 2. The isolated $\mathrm{WSe}_{2}$ islands often possess triangular shape, similar to what was earlier observed for $\mathrm{MoS}_{2}$ films [14]. The growth of spiral WSe 2 pyramids, $\sim 100$ times larger than in the Figure 2, has also been recently demonstrated [15]. Our STM measurements revealed that a significant $(80 \%)$ portion of nanostructures have their first atomic layer elevated by $2.4 \AA$. Such elevation (quasi-freestanding geometry) was earlier already reported for $\mathrm{WSe}_{2}$ films [16] and

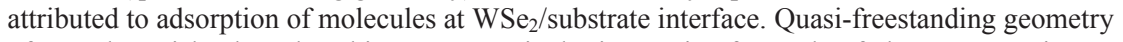
of monolayer islands makes this system particular interesting for study of phonon scattering and interference effects.

The typical STM image obtained on top of quasi-freestanding $1 \mathrm{ML} \mathrm{WSe}_{2}$ island is shown in the Figure 3. The size of this image is $190 \times 190 \AA^{2}$. The image was obtained at $35 \mathrm{pA}$ tunneling current and $1.7 \mathrm{~V}$ tunneling bias. The interference rings in this image can be clearly observed. The two types of patterns were observed: single-ring patterns (A-type patterns) and multi-ring (B-type) patterns. In the Figure 3, one of the A-type and one of the B-type patterns are schematically surrounded by dashed lines. For A-type patterns the interference ring has a diameter of $8.1 \AA$. For B-type patterns, the first ring also has a diameter of $8.1 \AA$; the second ring has a diameter of $19.2 \AA$; and the fragments of the third interference ring can also be seen in most of cases. The centers of the patterns correspond to interference minima having $0.2 \AA$ depths. The typical height of the first interference maxima is $0.1 \AA$. The absence of surface adatoms or other visible defects in the centers of these patterns indicates that the interference is

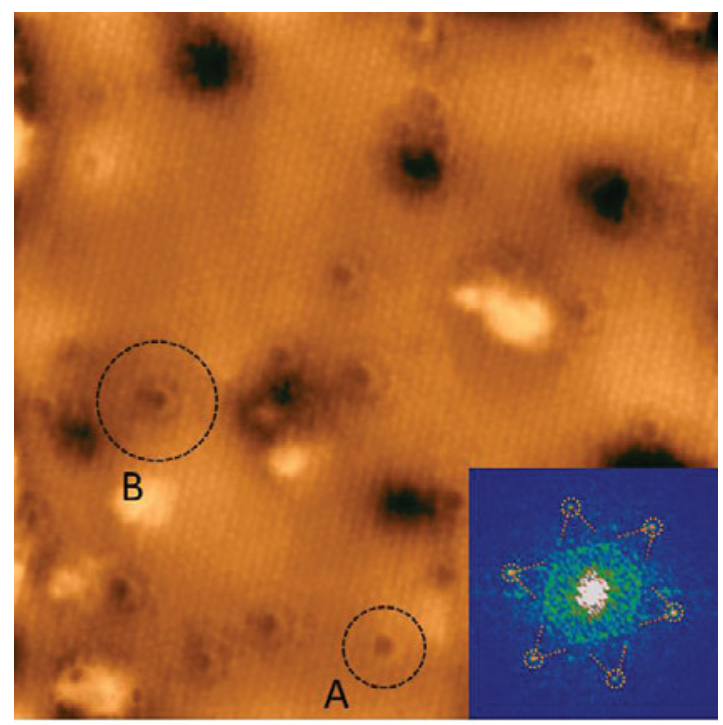

Figure 3. STM image obtained on top of quasi-freestanding $1 \mathrm{ML} \mathrm{WSe}_{2}$ island. The image size is $190 \times 190 \AA^{2}$. The two types of interference patterns can be clearly resolved on this image. One of the A-type and one of the B-type patterns are schematically surrounded by dashed lines. For B-type patterns, the first interference ring reveals symmetry breaking. Inset: 2D STM Fourier transform of the STM image reveals the 2D Brillouin zone. 
most likely induced by intercalating defects supporting $\mathrm{WSe}_{2}$ islands. The interference patterns do not depend on the tunneling bias: their centers always correspond to interference minima, and the separation between interference maxima always corresponds to $2 a_{0}$. Here $a_{0}=2.8 \AA$ is the spacing between neighboring atomic rows in $\mathrm{WSe}_{2}$ [17]. For 2D lattices $2 a_{0}$ represents the wavelength cutoff at the BZ edge, which determines the periodicity of phonon standing waves.

It makes sense to suggest that two different observed kinds of phonon interference patterns are related to two preferential attachment sites for intercalating defects, resulting in two different types of phonon scattering and synchronization regimes: strong synchronization for B-type patterns with large coherence radius (resonant scattering centers [18]), and weak synchronization for A-type patterns with short coherence radius (non-resonant scattering centers). The fact that the observed interference ring diameters correspond to $3 a_{0}$ and $7 a_{0}$ also confirms that scattering centers are located at higher-coordination inter-atomic sites. According to Ref. [19], STM observation of multiple high-coordination adsorption sites allows to identify these defects as adsorbed $\mathrm{CO}$ molecules. Intercalation by $\mathrm{CO}$ molecules can provide more robust boundary conditions for phonon interference, because of local binding between the islands and the substrate induced by their dipole moments. The interference patterns were only observed on elevated monolayer islands.

The additional insight into the origin of observed interference patterns can be obtained from the 2D Fourier analysis of STM images, shown in the inset of Figure 3. The six peaks from the hexagonal crystal lattice represent here the reciprocal lattice vectors $\pm 2 \pi / a_{0}$. The first BZ in this Figure can be found from the intersection of six Bragg planes (schematically shown by dotted lines). A large hexagonal disk in this Figure represents the area of $k$-space that contributes to interference. Essentially, this observation confirms the optical phonon amplitude summation across BZ previously discussed in the Figure 1, including sharp BZ edge. The analysis of STM images shows that for B-type patterns a visible angular segmentation of interference amplitude takes place: the inner rings show visible $180^{\circ}$ segmentation (symmetry breaking), whereas the outer rings show $60^{\circ}$ segmentation (less visible at higher atomic contrasts as in Figure 3). The missing portions of interference wave packets are most likely caused by phonon standing wave selection rule: interference maxima coinciding with inter-atomic sites become suppressed. As a result, each type of interference pattern is characterized by unique real-space distribution of allowed and suppressed interference maxima, in agreement with our data.

\section{DISCUSSION}

Development of phonon standing waves in quasi-freestanding geometry is rather anticipated effect. Our key experimental findings: correlation between interference signals and quasifreestanding geometry of islands, bias independent $2 a_{0}$ periodicity of fringes, produced by BZ edge, which we directly observe on Fourier images, combined with symmetry breaking effect, observation of two types of interference patterns, and $3 a_{0}$ vs. $7 a_{0}$ absolute ring diameters (a) do not have analogues among other known forms of nanoscale interference phenomena and (b) are naturally explained by phonon standing waves mechanism. We would also like to mention here that the alternative interference mechanism, electronic Friedel oscillations [20], as earlier STM studies [14, 21,22] have shown, is usually not manifested in monolayers of transition metal dichalcogenide (TMD) semiconductors, probably due to its suppression caused by lack of inversion symmetry [23]. 
The possibility of detecting phonon standing waves using STM requires separate clarification. Detection of phonon oscillations in STM experimental setup may be attributed to exponential distance dependence of electronic matrix elements and electron-phonon coupling [9]. The exponential distance dependence of tunneling current represents one of such rectification mechanisms. For example, for out-of-plane atomic oscillations tunneling into positive (outwards directed) half-periods is anticipated to be exponentially larger than for negative (inwards directed) half-periods. This may result in net increase of tunneling current accompanied by retraction of the STM tip. Assuming zero anharmonicity of atomic motion,

STM signals would be $z \propto A^{2} / 4 \lambda$, where $\lambda$ is vacuum decay length for tunneling current $\sim 0.4 \AA$. For anticipated oscillation amplitudes of $0.05-0.1 \AA$ the signals would be below STM resolution level. A significantly different $z(A)$ dependence is expected for strongly asymmetric anharmonic atomic motion. In this case, due to asymmetric oscillator probability distribution the STM signals would be $z \propto A$, and efficient STM detection of phonon standing waves would be anticipated. In the STM experiment, the required asymmetry can be induced by asymmetric electronic response, and does not have to be present in atomic motion itself. Such electronic anharmonicity manifests due to phonon-induced modulation of W-Se bonds [24], and this effect may include contributions of both out-of-plane $\left(\mathrm{A}_{1}^{\prime}\right)$ and in-plane ( $\left.E^{\prime}\right)$ phonon modes. We also cannot exclude the possibility that some contribution to STM contrast of interference patterns arises from static atomic displacements produced by phonon synchronization effect, as it was discussed in Ref. [8].

Unlike in the simplest one-dimensional case that was discussed in the Figure 1, in two dimensions the summation across BZ includes $\propto k$ increase of the phase space area and $k$ dependent phonon scattering cross section $\sigma(k)$. Interestingly, the product of $k$ and $\sigma(k)$ is anticipated to be constant for 2D resonant Breit-Wigner scattering $[18,25,26]$. This explains why for B-type defects the profile of the interference pattern strongly resembles 1D simulation from the Figure 1. For A-type defects the scattering is probably non-resonant.

Simple estimations show that at cryogenic temperatures optical phonon amplitudes can decrease by $25 \%$ and even at our present measurement conditions quantum zero-point oscillations significantly contribute to the interference contrast.

\section{CONCLUSIONS}

The overall data analysis strongly indicates that phonon interference has been observed in our STM experiments, most likely due to enhancement of signals facilitated by quasifreestanding geometry of islands. This phenomenon opens new experimental pathway for study of deformation-induced electronic structure changes, phonon-stimulated intervalley transitions, and defect-induced interactions in $2 \mathrm{D}$ semiconductors.

\section{ACKNOWLEDGEMENTS}

The authors thank K. A. Matveev, N. Zhitenev, M. Bode, A. V. Balatsky, Ya. B. Bazaliy, A. L. Chernyshev, I. Zaliznyak, S. Mou, P. Nikolaev, M. Check, A. Safriet, Y. C. Lin, M. McConney, R. Rao and A. N. Reed for help and interesting discussions. The work at Penn State was supported by the Center for Low Energy Systems Technology (LEAST), one of six centers supported by the STARnet phase of the Focus Center Research Program (FCRP), a 
Semiconductor Research Corporation (SRC) program sponsored by MARCO and DARPA. Financial support from the Air Force Office of Scientific Research is gratefully acknowledged.

\section{REFERENCES}

* e-mail Igor.Altfeder.Ctr@us.af.mil

1. A. Fasolino, J. H. Los, and M. I. Katsnelson, Nature Materials 6, 858 (2007)

2. J. Fransson and A. V. Balatsky, Phys. Rev. B 75, 195337 (2007)

3. I. Altfeder, A. A. Voevodin, and A. K. Roy, Phys. Rev. Lett. 105, 166101 (2010)

4. A. Balandin and K. L. Wang, Phys. Rev. B 58, 1544 (1998)

5. J. F. Rodriguez-Nieva, R. Saito, S. D. Costa, and M. S. Dresselhaus, Phys. Rev. B 85, 245406

6. M. V. Klein, Phys. Rev. 131, 1500 (1963)

7. G. Manzano, F. Galve, G. L. Giorgi, E. Hernández-García, and R. Zambrini, Sci. Rep. 3, 1439 (2013)

8. Yu. N. Gornostyrev, M. I. Katsnelson, A. P. Platonov, A. V. Trefilov, JETP 80, 525 (1995)

9. O. Rösch, O. Gunnarsson, Phys. Rev. Lett. 92, 146403 (2004)

10. M. Boukhicha, M. Calandra, M. A. Measson, O. Lancry, and A. Shukla, Phys. Rev. B 87, 195316 (2013)

11. H. Sahin, S. Tongay, S. Horzum, W. Fan, J. Zhou, J. Li, J. Wu and F. M. Peeters, Phys. Rev. $B$ 87, 165409 (2013)

12. S. M. Eichfeld, C. M. Eichfeld, Y. C. Lin, L. Hossain, and J. A. Robinson, APL Mat. 2, 092508 (2014)

13. S. M. Eichfeld et al., ACS Nano 9, 2080 (2015)

14. C. Zhang, A. Johnson, C. L. Hsu, L. J. Li, and C. K. Shih, Nano Lett. 14, 2443 (2014)

15. L. Chen, B. Liu, A. N. Abbas, Y. Ma, X. Fang, Y. Liu, and C. Zhou, ACS Nano 8, 11543 (2014)

16. P. Tonndorf et al., Opt. Express 21, 4908 (2013)

17. J. A. Wilson, A. D. Yoffe, Adv. Phys. 18, 193 (1969)

18. J. H. Crawford and L. M. Slifkin, Point Defects in Solids: Volume 2, Semiconductors and Molecular Crystals, Plenum Press, New York, 1975

19. Q. Yue, Z. Shao, S. Chang and J. Li, Nanoscale Research Letters 8, 425( 2013)

20. M. F. Crommie, C. P. Lutz, and D. M. Eigler, Nature 363, 524 (1993)

21. M. V. Bollinger, J. V. Lauritsen, K. W. Jacobsen, J. K. Nørskov, S. Helveg, and F.

Besenbacher, Phys. Rev. Lett. 87, 196803 (2001)

22. M. M. Ugeda et al., Nature Materials 13, 1091 (2014)

23. L. Zhang, K. Liu, A. B. Wong, J. Kim, X. Hong, C. Liu, T. Cao, S. G. Louie, F. Wang, and P. Yang, Nano Lett. 14, 6418 (2014)

24. C. H. Chang, X. Fan, S. H. Lin, and J. L. Kuo, Phys. Rev. B 88, 195420 (2013)

25. L. Chaos-Cador and G. Garcia-Calderon, J. Phys. A: Math. Theor. 43, 035301 (2010)

26. L. D. Landau and E. M. Lifshitz, Quantum Mechanics, Pergamon, London, 1958 